\title{
Wavelength Selection by Interrupted Coarsening in Reaction-Diffusion Systems
}

\author{
Fridtjof Brauns $\odot,{ }^{1,}{ }^{*}$ Henrik Weyer $\odot,{ }^{1}$ Jacob Halatek, ${ }^{2}$ Junghoon Yoon $\odot,{ }^{1}$ and Erwin Frey $\oplus^{1, \dagger}$ \\ ${ }^{1}$ Arnold Sommerfeld Center for Theoretical Physics and Center for NanoScience, Department of Physics, \\ Ludwig-Maximilians-Universität München, Theresienstraße 37, D-80333 München, Germany \\ ${ }^{2}$ Biological Computation Group, Microsoft Research, Cambridge CB1 2FB, United Kingdom
}

(Received 5 May 2020; revised 19 October 2020; accepted 9 December 2020; published 9 March 2021)

Wavelength selection in reaction-diffusion systems can be understood as a coarsening process that is interrupted by counteracting processes at certain wavelengths. We first show that coarsening in massconserving systems is driven by self-amplifying mass transport between neighboring high-density domains. We derive a general coarsening criterion and show that coarsening is generically uninterrupted in two-component systems that conserve mass. The theory is then generalized to study interrupted coarsening and anticoarsening due to weakly broken mass conservation, providing a general path to analyze wavelength selection in pattern formation far from equilibrium.

DOI: 10.1103/PhysRevLett.126.104101

To predict the wavelength of patterns in highly nonlinear systems is a critical open problem, as wavelength selection is ubiquitous in a large range of nonequilibrium systems [1-7]. While the amplitude equation formalism and weakly nonlinear analysis have been highly successful in the vicinity of onset [8], these approaches are not informative for large amplitude patterns far away from onset. For onecomponent systems, a theory for wavelength selection based on a multiple-scale analysis has been developed $[9,10]$, but generalizations to multicomponent systems have remained elusive.

In this Letter, we propose that wavelength selection in reaction-diffusion systems can be understood as a coarsening process that is interrupted and even reversed by counteracting processes at certain wavelengths. Specifically, we study two-component systems and develop a theory for the mass-conserving case first where coarsening is uninterrupted. We then generalize this theory to account for source terms that break mass conservation and counteract the coarsening process.

While coarsening is well understood as minimization of the free energy for systems relaxing to thermal equilibrium (such as binary mixtures $[11,12]$ ), this reasoning is generally not applicable for nonequilibrium systems such as most reaction-diffusion systems. Two-component massconserving reaction-diffusion (MCRD) systems serve as paradigmatic models for intracellular pattern formation

Published by the American Physical Society under the terms of the Creative Commons Attribution 4.0 International license. Further distribution of this work must maintain attribution to the author(s) and the published article's title, journal citation, and DOI.
[13-19] and are used as phenomenological models for a wide range of systems including precipitation patterns [20], granular media [21], and braided polymers [22]. It has long been speculated that two-component MCRD systems generically exhibit uninterrupted coarsening [16,19,23,24]. However, it has remained unclear whether coarsening always goes to completion in two-component MCRD systems, largely owing to a lack of insight into the underlying physical processes.

Here, we show that coarsening is driven by positive feedback in the competition for mass, derive a simple and quantitative description of the coarsening dynamics, and explain why coarsening is generically uninterrupted in two-component MCRD systems. As they are grounded in a phase-space analysis [25], our results are independent of the specific mathematical form of the reaction kinetics.

Building on the insights into the coarsening process in the mass-conserving case, we elucidate and quantify the physical mechanisms underlying wavelength selection in the presence of weak source terms (weakly broken mass conservation). Coarsening arrests when mass competition is balanced by production and degradation. Moreover, domain splitting-owing to the destabilization of plateaus-reverses coarsening. Both are graphically understood by a generalization of the phase-space analysis. Since our approach builds on studying the spatial redistribution of a nearly conserved quantity, we expect that it can be generalized beyond two-component reactiondiffusion systems, for instance, to systems with more components and to hydrodynamic models for active matter systems [3,26-29].

The general form of a reaction-diffusion system with two components, $u$ and $v$, can be written as 


$$
\begin{aligned}
& \partial_{t} u(x, t)=D_{u} \nabla^{2} u+f(u, v)+\varepsilon s_{1}(u, v), \\
& \partial_{t} v(x, t)=D_{v} \nabla^{2} v-f(u, v)+\varepsilon s_{2}(u, v),
\end{aligned}
$$

on a domain $\Omega$, with either no-flux or periodic boundary conditions [30]. For specificity, we choose $D_{u}<D_{v}$ [31]. The reaction term $f$ describes conversion between $u$ and $v$, while the source terms $s_{1,2}$ with a (small) dimensionless source strength $\varepsilon$ break mass conservation.

Let us first analyze the mass-conserving case $\varepsilon=0$. Then, the total density $\rho=u+v$ is conserved such that the average $\bar{\rho}=|\Omega|^{-1} \int_{\Omega} d x \rho(x, t)$ remains constant. The time evolution of $\rho$ is given by $[13,22,23,25]$

$$
\partial_{t} \rho(x, t)=D_{v} \nabla^{2} \eta(x, t)
$$

with the mass-redistribution potential defined by $\eta:=v+\left(D_{u} / D_{v}\right) u$; the corresponding dynamical equation for $\eta(x, t)$ is given in Supplemental Material [32], Sec. 1.1. For stationary patterns $[\tilde{u}(x), \tilde{v}(x)]$, the mass-redistribution potential must be spatially uniform, $\eta(x)=\eta_{\text {stat }}$. Based on this, one can analyze two-component MCRD systems in the $(u, v)$ phase plane [25]: There, stationary patterns are constrained to a linear subspace, $v+\left(D_{u} / D_{v}\right) u=\eta_{\text {stat }}$, called flux-balance subspace (FBS); see Fig. 1(b). The intercept $\eta_{\text {stat }}$ is determined by the balance of the spatially integrated reactive flows (total turnover balance), corresponding (approximately) to a balance of areas [shaded in red in Fig. 1(b)] enclosed by the FBS and the reactive nullcline $(f=0, \mathrm{NC})$. The FBS-NC intersection points

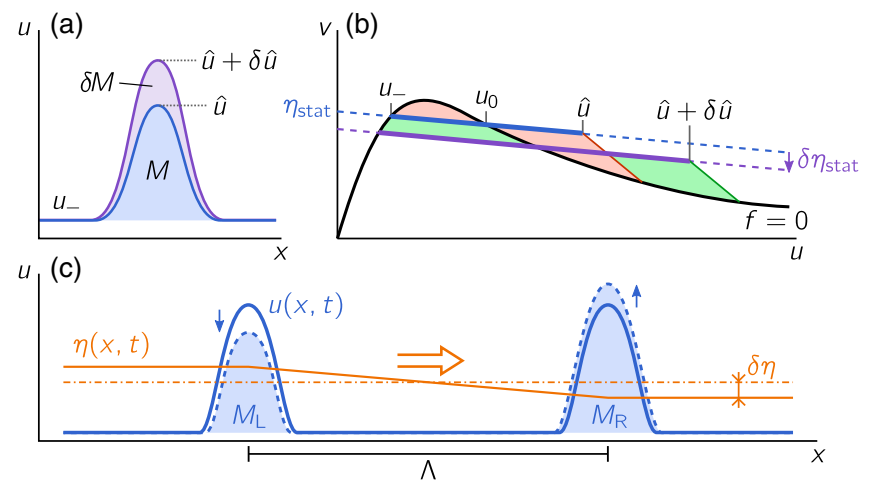

FIG. 1. (a) Illustration of a stationary peak with peak mass $M$. Increasing the mass to $M+\delta M$ increases the peak amplitude to $\hat{u}+\delta \hat{u}$. (b) Representation of the stationary peak in phase space (thick blue line), which is constrained to the FBS (dashed blue line). The FBS offset $\eta_{\text {stat }}(M)$ is determined by a balance of total reactive turnovers (areas shaded in red). For a peak with increased mass $M+\delta M$, and thus increased peak amplitude $\delta \hat{u}$, the FBS shifts downward $\delta \eta_{\text {stat }}$ until total turnover balance is restored (balance of green-shaded areas). (c) After a perturbation of two identical stationary peaks, the gradient in the mass-redistribution potential $\eta$ (orange line) drives mass transport between the peaks (orange arrow) such that the larger (smaller) peak grows (shrinks) further (blue arrows). correspond to the plateau(s) and inflection point(s) of a stationary pattern. Two types of patterns can be distinguished-mesas and peaks. The elementary mesa pattern is composed of two plateaus, connected by an interface (or "kink"), while a peak forms when the maximum density does not saturate in a high-density plateau [Fig. 1(a); compare Fig. 2(a)] [25,47]. We begin the analysis with peak patterns and then generalize the results to mesas.

A mass-competition instability drives coarsening.Coarsening requires the transport of mass between peaks. Because mass transport is diffusive, it is fastest on the shortest length scales; hence, the dominant process is competition for mass between neighboring peaks [Fig. 1(a)]. Thus, as an elementary case, we study two peaks in a "box" with no-flux boundary conditions. Consider a situation ("coarsening limit") where the peaks are well separated, such that diffusive transport is limiting. We can then approximate the peaks to be in (regional) quasisteady state (QSS), such that $\eta=\eta_{\text {stat }}(M)$ at a given peak with total mass $M$. This approximation is commonly applied in thin film theory [48,49] and Ostwald ripening $[11,12]$.

Starting from two identical, stationary peaks, each with total mass $M_{0}$, the dynamics of the mass difference between them $\left(M_{R, L}=M_{0} \pm \delta M\right)$-obtained by integration of Eq. (2) over a single peak-is determined by the $\eta$ gradients in the plateau between them [indicated by the orange arrow in Fig. 1(c)]. Using QSS at each peak separately, the mass-redistribution potential at the peaks is given by $\eta_{R, L}=\eta_{\text {stat }} \pm\left(\left.\partial_{M} \eta_{\text {stat }}\right|_{M_{0}}\right) \delta M$. Between the peaks, $\eta$ obeys $\partial_{x}^{2} \eta=0$, because diffusive relaxation within the plateau is fast compared to the peak evolution (see Supplemental Material Sec. 2 [32] for details). Thus, in 1D, the resulting gradient in $\eta$ is linear and determined by $\eta=\eta_{R, L}$ at the peak positions. For a given peak separation $\Lambda$, this approximation determines the dynamics of mass redistribution

$$
\partial_{t} \delta M \approx-\frac{2 D_{v}}{\Lambda}\left(\left.\partial_{M} \eta_{\text {stat }}\right|_{M_{0}}\right) \delta M=: \sigma_{D} \delta M
$$

The subscript $D$ denotes the diffusion-limited regime. If the growth rate $\sigma_{D}$ is positive, an instability driven by positive feedback in competition for mass results in coarsening. Hence, the condition for uninterrupted coarsening reads

$$
\partial_{M} \eta_{\text {stat }}(M)<0
$$

i.e., that $\eta_{\text {stat }}(M)$ is a strictly monotonically decreasing function for all stable stationary single-peak solutions. This recovers a previous, mathematically derived coarsening condition [13,23]. Importantly, the analysis presented here gives insight into the underlying physical mechanism and shows that not only the criterion for coarsening, but the entire temporal evolution of coarsening is determined by $\partial_{M} \eta_{\text {stat }}$ via Eq. (3) [50]. We learn that the functional 
dependence of the mass-redistribution potential on the peak mass, $\eta_{\text {stat }}(M)$, plays a role analogous to the functional dependence of the chemical potential on the droplet size that drives Ostwald ripening or to the film height in dependence of droplet size that drives coarsening of unstable thin films $[48,49]$.

Generic coarsening laws for mass-conserving systems.-To show that coarsening is uninterrupted, we need to show that the criterion Eq. (4) holds and continues to hold as small peaks disappear, causing the mass of the remaining peaks to increase. For an intuitive argument, consider a single stationary peak with mass $M$ [see Fig. 1(a)] and its representation in phase space, the blue line in Fig. 1(b). Add an amount $\delta M$ of mass and hold $\eta_{\text {stat }}$ fixed for the moment (for the sake of argument). Fixing $\eta_{\text {stat }}$ also fixes the plateau $u_{-}$. Therefore, the additional mass will increase the peak amplitude $\hat{u}$ [Fig. 1(b)], causing the reactive turnover to the right of $u_{0}$ to increase. The resulting imbalance of total turnover entails a net reactive flow that shifts the flux-balance subspace downward, i.e., lowers $\eta_{\text {stat }}$, to restore total turnover balance. We conclude that $\eta_{\text {stat }}(M)$ is generically a monotonically decreasing function. (More rigorous arguments are given in Supplemental Material Secs. 4 and 5 [32]).

Let us now turn to the dynamic coarsening laws. As an example, consider $f_{\mathrm{ex}}=(1+u) v-u /(1+u)$, where the first and second terms may, for instance, describe protein recruitment and first-order enzymatic detachment, respectively. A simple scaling argument [51] yields a power-law relation $\eta_{\text {stat }}(M) \sim M^{-\alpha}$, where the exponent depends on the specific reaction kinetics $(\alpha=2 / 3$ for the example above); see Fig. 2(b). In a large system containing multiple peaks, the average peak separation $\langle\Lambda\rangle$ is linked to the characteristic peak mass by $\langle M\rangle=\left(\bar{\rho}-\rho_{-}\right)\langle\Lambda\rangle$, where $\rho_{-}$ is the total density in the low-density plateau between the peaks and $\langle\cdot\rangle$ denotes an average over the entire system. As peaks collapse, with a typical time given by the inverse growth rate of the mass-competition instability $t \sim \sigma_{D}^{-1}$, the average peak separation $\langle\Lambda\rangle$ will increase.
Combining $\sigma_{D} \sim-\left\langle\partial_{M} \eta_{\text {stat }}\right\rangle /\langle\Lambda\rangle$ with $\left\langle\partial_{M} \eta_{\text {stat }}\right\rangle \sim\langle M\rangle^{-\alpha-1} \sim$ $(\bar{\rho}\langle\Lambda\rangle)^{-\alpha-1}$ yields power-law coarsening with $\langle\Lambda\rangle(t) \sim$ $t^{1 /(2+\alpha)}$; see Fig. 2(c) and Fig. S4 [32]. Moreover, using appropriate scaling amplitudes, the coarsening trajectories for different average masses $\bar{\rho}$ can be collapsed onto a single master curve obtained from $\partial_{M} \eta_{\text {stat }}$ (see Supplemental Material Sec. 3 [32]). Power-law coarsening in 1D has previously been found for peaklike droplets formed during the dewetting of thin liquid films [48].

As peaks collapse, those remaining grow in mass and height. When the density at the peak maximum saturates in a high-density plateau (corresponding to a FBS-NC intersection point in phase space), a mesa pattern starts to form [Fig. 2(a) and Fig. S3 [32]] [52]. For such mesas, somewhat more subtle arguments show that $\eta_{\text {stat }}(M)$ remains a monotonically decreasing function (see Supplemental Material Sec. 5 [32]). In essence, changing $M$ shifts the interface positions and, thus, changes the width of a mesa's plateau. As the density profile approaches the limiting plateaus $u_{ \pm}\left(\eta_{\text {stat }}^{\infty}\right)$ through exponential tails, $\eta_{\text {stat }}(M)$ approaches $\eta_{\text {stat }}^{\infty}$ exponentially slowly [see the inset in Fig. 2(b)], where we define $\eta_{\text {stat }}^{\infty}$ as the limit of $\eta_{\text {stat }}$ for the stationary pattern on an infinite domain (see Supplemental Material Sec. 5.1 [32]). Using the same scaling arguments as for peaks, one obtains a logarithmic coarsening law for all mesa patterns, as in the one-dimensional Cahn-Hilliard model [53]. For the concrete example $f_{\mathrm{ex}}$, we find excellent agreement between finite-element simulations and $\langle\Lambda\rangle(t)$ obtained from $\eta_{\text {stat }}(M)$ by these scaling arguments [see Fig. 2(c)]. Based on the physical insights presented above, a generalization to more than one spatial dimension is straightforward. For mesalike droplets with radius $R$, one finds $\eta_{\text {stat }}-\eta_{\text {stat }}^{\infty} \sim R^{-1}$, which yields power-law coarsening with the universal exponent $1 / 3$ (see Supplemental Material Sec. 5.4 [32]). For peaklike droplets, we expect system-dependent exponents as in 1D.

The limit of large $D_{v}$. - For $D_{v} \rightarrow \infty$, mass redistribution by $v$ diffusion becomes instantaneous, such that the reactive conversion between $u$ and $v$, which drives the (a)

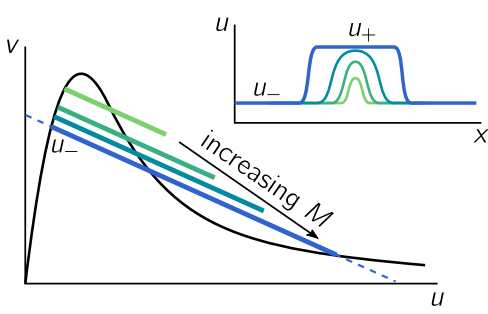

(b)

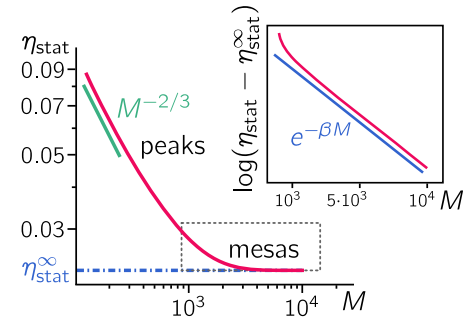

(c)

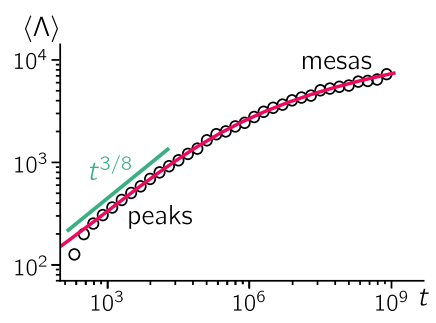

FIG. 2. (a) Illustration of the peak to mesa transition as the total mass $M$ is increased. (b) The function $\eta_{\text {stat }}(M)$ obtained by numerical continuation of the stationary solutions for the reaction kinetics $f_{\text {ex }}$. Crossover from power law for peak patterns (amplitude not saturated) to exponential approach to $\eta_{\text {stat }}^{\infty}$ for mesa patterns. (c) Coarsening dynamics from finite element simulations for $f_{\text {ex }}$ (black circles; mean peak distance averaged over four independent runs started from random initial conditions; parameters: $D_{u}=1, D_{v}=10^{4}$, $\bar{\rho}=1.5$, and system size $|\Omega|=2 \times 10^{5}$, periodic boundary conditions). The red line shows the analytic prediction based on $\sigma_{D}$ from $\eta_{\text {stat }}(M)$, shown in (b), via Eq. (3). After an initial transient, power-law coarsening $\Lambda \sim t^{3 / 8}$ for peaks is observed, which flattens into logarithmic coarsening for mesas. 
growth or shrinking of mesas or peaks, becomes limiting. In this reaction-limited case, we find $\sigma_{R} \approx\left(\partial_{M} \eta_{\text {stat }}\right)$ $\ell_{\text {int }}\left\langle f_{v}\right\rangle_{\text {int }}$, where $\ell_{\text {int }}$ is the interface width and $\langle\cdot\rangle_{\text {int }}$ denotes the average over the interface region (see Supplemental Material Sec. 6 [32] for details and numerical verification). Comparing with Eq. (3) shows that the coarsening criterion Eq. (4) holds in both regimes, and the crossover from diffusion- to reaction-limited coarsening occurs at $D_{v} / \Lambda \approx \ell_{\text {int }}\left\langle f_{v}\right\rangle_{\text {int }}$.

Weakly broken mass conservation.-With an understanding for the coarsening dynamics in the strictly mass-conserving system, we now consider the effect of slow production and degradation for $0<\varepsilon \ll 1$. We will see that these additional processes interrupt coarsening $[1,2,26,54]$ and can reverse it by inducing peak and mesa splitting [5,7,55], thus selecting a range of stable pattern wavelengths. In the presence of source terms, the time evolution of the total density $\rho$ is governed by

$$
\partial_{t} \rho=D_{v} \partial_{x}^{2} \eta+\varepsilon s(u, v)
$$

with the total source $s:=s_{1}+s_{2}$. Hence, the average mass $\langle\rho\rangle$ is no longer a control parameter but a time-dependent variable that is determined indirectly by a balance of production and degradation (in short, source balance). In phase space, there are now two reactive nullclines, one each for $u$ and $v$, which both converge to $f=0$ for $\varepsilon \rightarrow 0$. Their intersection point(s) determine(s) the homogeneous steady state (HSS) $\rho_{\text {HSS }}$ that balances the total source term.

In the following, we restrict ourselves to mesa patterns. To lowest order in $\varepsilon$, source balance determines the "half lengths" $L_{ \pm}$of the upper and lower plateaus (see Supplemental Material Sec. 7 [32]). Along the plateaus, the spatial gradients induced by slow production or degradation ( $\varepsilon$ small) are shallow, such that the dynamics is (approximately) slaved to the nullcline $f=0$ [see Fig. 3(b)]. This justifies a local equilibrium approximation $s(u, v) \approx s\left[u^{*}(\rho), v^{*}(\rho)\right] \equiv s^{*}(\rho)$ in Eq. (5), where the local equilibria are defined by $f\left(u^{*}, v^{*}\right)=0$ and $u^{*}+v^{*}=\rho$. On the short scale of the interface width, the weak source term is negligible, and each interface constrained to a fluxbalance subspace. We are now in a position to generalize the phase-space analysis introduced in Ref. [25] and analyze interrupted coarsening and mesa splitting.

(i) Peak and mesa splitting. - Consider the fully coarsened state for $\varepsilon=0$ and add a small source term such that $s^{*}\left(\rho_{+}\right)<0$ and $s^{*}\left(\rho_{-}\right)>0$ [i.e., $\rho_{-}<\rho_{\mathrm{HSS}}<\rho_{+}$; see Fig. 3(b)] [56]. The upper plateau is depressed by net degradation and is refilled by inflow from the interfaces that connect to the lower plateau where net production prevails. The longer the plateaus (and the larger $\varepsilon$ ), the more they curve toward $\rho_{\mathrm{HSS}}$. Since $\rho_{-}<\rho_{\mathrm{HSS}}<\rho_{+}, \quad \rho(x)$ will eventually enter the interval of lateral instability $\left[\rho_{\text {lat }}^{-}, \rho_{\text {lat }}^{+}\right]$ (where $\partial_{\rho} \eta^{*}<0$ ), triggering a nucleation event that results in the splitting of the mesa [see Fig. 3(a) and Movie 2 [32] ]. A simple approximation for the threshold wavelength $\Lambda_{\text {split }}(\varepsilon)$ where this happens is derived in Supplemental Material, Sec. 7.1 [32]. Comparison with numerical simulations shows excellent agreement [see Fig. 3(d)].

(ii) Interrupted coarsening.-Intuitively, production and degradation can counteract the mass-competition instability. To determine the corresponding length scale $\Lambda_{\text {stop }}$ where coarsening arrests, we consider the stability of two neighboring, symmetric mesas. A perturbation that moves a small amount of mass from one mesa to the other [Fig. 3(c)] has two effects: First, it shifts the mass-redistribution potential at the interfaces, leading to mass transport that further amplifies the perturbation with rate $\sigma_{D}(\Lambda) \delta M$ as in the strictly mass-conserving situation; cf. Eq. (3). Second, the changed lengths $\delta L=$ $\delta M /\left(\rho_{+}-\rho_{-}\right)$of the two mesas result in net production (degradation) in the shorter (longer) mesa with rate $\varepsilon\left|s^{*}\left(\rho_{\text {outer }}\right)\right| \delta L$ [indicated by the purple arrows in Fig. 3(c)].
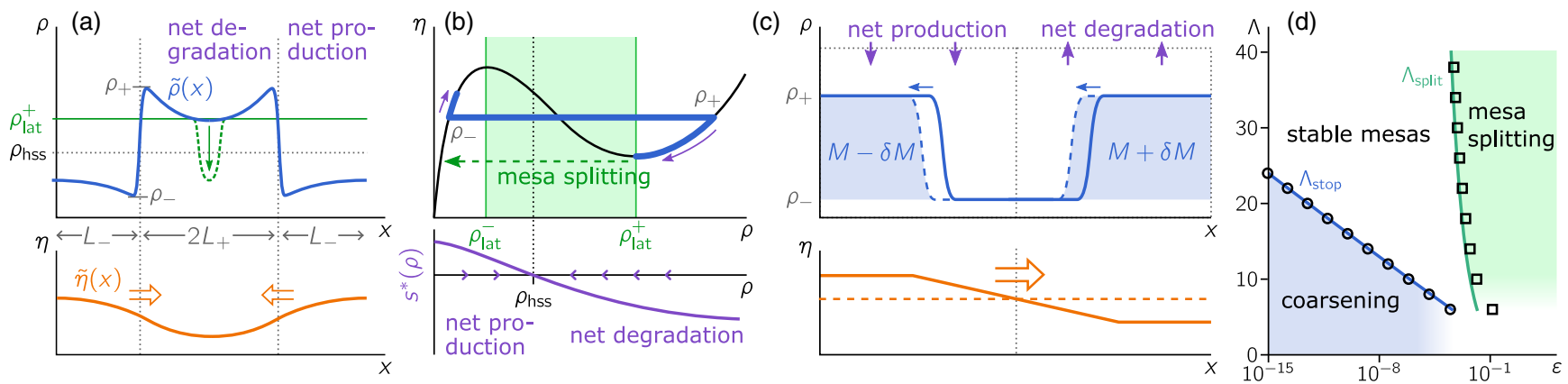

FIG. 3. Wavelength selection by weakly broken mass conservation. (a),(b) Mesa splitting: (a) real space profiles of $\tilde{\rho}(x)$ and $\tilde{\eta}(x)$ and (b) phase space in $(\rho, \eta)$ coordinates, with the source term in local equilibrium approximation plotted below. The green shaded area indicates the region of lateral instability. (c) Interrupted coarsening due to a balance of production, degradation, and mass redistribution between neighboring mesas. (d) Regimes separated by interrupted coarsening (circles) and mesa splitting (squares) as well as analytic approximations (blue and green lines) for large $\Lambda$ and small $\varepsilon$. While in the coarsening regime (blue) stationary patterns are unstable, no stationary patterns exist in the mesa-splitting regime (green). In the regime of small $\Lambda$ and large $\varepsilon$, corrections become large and the approximations do not hold (see Supplemental Material Sec. 7 [32]). Parameters: $D_{u}=0.1, D_{v}=1$, and $p=2$. 
Here $\rho_{\text {outer }}$ denotes the total density of the outer plateau [the inner plateau shifts as a whole and does not change in length; see Fig. 3(c)]. Together, the balance of both processes determines $\Lambda_{\text {stop }}$ (see Supplemental Material Sec. 7.2 [32] for details)

$$
\sigma_{D}\left(\Lambda_{\text {stop }}\right) \approx \varepsilon \frac{\left|s^{*}\left(\rho_{\text {outer }}\right)\right|}{\rho_{+}-\rho_{-}}
$$

As a concrete example, we apply Eq. (6) to the "Brusselator" model [57] $\left(f=u^{2} v-u, s=p-u\right)$ and find excellent agreement with numerics [Fig. 3(d)]. Notably, the simple estimate given by Eq. (6) generalizes previous, mathematically obtained results $[54,58]$.

Our analysis shows that the mechanisms underlying mesa splitting and interrupted coarsening are distinct. In particular, the length scale where coarsening stops is much smaller than the length scale where mesas and peaks split [see Fig. 3(d)]. This implies that there are stable periodic patterns for a large, continuous range of wavelengths (multistability), as was shown previously for the Brusselator [54,55,57]. Similarly, multistability of wavelengths was recently found in a hydrodynamic model for flocking [3]. Interestingly, a unique length scale is selected once noise is accounted for [4]. Noise-driven wavelength selection was also observed in an "active model B" [59]. It would be interesting to study whether this phenomenon is also found in reaction-diffusion systems.

Another interesting open problem are systems with cross diffusion and density-dependent diffusion coefficients (see, e.g., Refs. [60-63]). We also expect that our approach can be generalized to systems with more than two components, higher spatial dimensions, and also beyond reaction-diffusion systems. In particular, conserved densities (particle numbers) are a generic feature of many active matter systems in which coarsening and length-scale selection ("microphase separation") are of growing interest [3,26-29,59,64-70].

This work was funded by the Deutsche Forschungsgemeinschaft (DFG, German Research Foundation) through the Collaborative Research Center (SFB) 1032-ProjectID No. 201269156 - and the Excellence Cluster ORIGINS under Germany's Excellence Strategy-EXC-2094390783311.

F. B. and H. W. contributed equally to this work.

*fridtjof.brauns@lmu.de †rey@lmu.de

[1] F. Liu and N. Goldenfeld, Dynamics of phase separation in block copolymer melts, Phys. Rev. A 39, 4805 (1989).

[2] S.C. Glotzer, E. A. Di Marzio, and M. Muthukumar, Reaction-Controlled Morphology of Phase-Separating Mixtures, Phys. Rev. Lett. 74, 2034 (1995).
[3] J.-B. Caussin, A. Solon, A. Peshkov, H. Chaté, T. Dauxois, J. Tailleur, V. Vitelli, and D. Bartolo, Emergent Spatial Structures in Flocking Models: A Dynamical System Insight, Phys. Rev. Lett. 112, 148102 (2014).

[4] A. P. Solon, H. Chaté, and J. Tailleur, From Phase to Microphase Separation in Flocking Models: The Essential Role of Nonequilibrium Fluctuations, Phys. Rev. Lett. 114, 068101 (2015).

[5] S. M. Murray and V. Sourjik, Self-organization and positioning of bacterial protein clusters, Nat. Phys. 13, 1006 (2017).

[6] J.-g. Chiou, K. D. Moran, and D. J. Lew, How cells determine the number of polarity sites, bioRxiv https:// doi.org/10.1101/2020.05.21.109520 (2020).

[7] C. Gai, D. Iron, and T. Kolokolnikov, Localized outbreaks in an S-I-R model with diffusion, J. Math. Biol. 80, 1389 (2020).

[8] M. C. Cross and P. C. Hohenberg, Pattern formation outside of equilibrium, Rev. Mod. Phys. 65, 851 (1993).

[9] P. Politi and C. Misbah, When Does Coarsening Occur in the Dynamics of One-Dimensional Fronts? Phys. Rev. Lett. 92, 090601 (2004).

[10] P. Politi and C. Misbah, Nonlinear dynamics in one dimension: A criterion for coarsening and its temporal law, Phys. Rev. E 73, 036133 (2006).

[11] C. Wagner, Theorie der Alterung von Niederschlägen durch Umlösen (Ostwald-Reifung), Z. Elektrochem. 65, 581 (1961).

[12] I. Lifshitz and V. Slyozov, The kinetics of precipitation from supersaturated solid solutions, J. Phys. Chem. Solids 19, 35 (1961).

[13] M. Otsuji, S. Ishihara, C. Co, K. Kaibuchi, A. Mochizuki, and S. Kuroda, A mass conserved reaction-diffusion system captures properties of cell polarity, PLoS Comput. Biol. 3, e108 (2007).

[14] A. B. Goryachev and A. V. Pokhilko, Dynamics of Cdc42 network embodies a Turing-type mechanism of yeast cell polarity, FEBS Lett. 582, 1437 (2008).

[15] S. J. Altschuler, S. B. Angenent, Y. Wang, and L. F. Wu, On the spontaneous emergence of cell polarity, Nature (London) 454, 886 (2008).

[16] Y. Mori, A. Jilkine, and L. Edelstein-Keshet, Wave-pinning and cell polarity from a bistable reaction-diffusion system, Biophys. J. 94, 3684 (2008).

[17] A. Jilkine and L. Edelstein-Keshet, A comparison of mathematical models for polarization of single eukaryotic cells in response to guided cues, PLoS Comput. Biol. 7, e1001121 (2011).

[18] P. K. Trong, E. M. Nicola, N. W. Goehring, K. V. Kumar, and S. W. Grill, Parameter-space topology of models for cell polarity, New J. Phys. 16, 065009 (2014).

[19] J.-G. Chiou, S. A. Ramirez, T. C. Elston, T. P. Witelski, D. G. Schaeffer, and D. J. Lew, Principles that govern competition or co-existence in Rho-GTPase driven polarization, PLoS Comput. Biol. 14, e1006095 (2018).

[20] A. Scheel, Robustness of Liesegang patterns, Nonlinearity 22, 457 (2009).

[21] I. Aranson and L. Tsimring, Granular Patterns (Oxford University Press, New York, 2008).

[22] G. Forte, M. Caraglio, D. Marenduzzo, and E. Orlandini, Plectoneme dynamics and statistics in braided polymers, Phys. Rev. E 99, 052503 (2019). 
[23] S. Ishihara, M. Otsuji, and A. Mochizuki, Transient and steady state of mass-conserved reactiondiffusion systems, Phys. Rev. E 75, 015203(R) (2007).

[24] Y. Morita and T. Ogawa, Stability and bifurcation of nonconstant solutions to a reaction-diffusion system with conservation of mass, Nonlinearity 23, 1387 (2010).

[25] F. Brauns, J. Halatek, and E. Frey, Phase-Space Geometry of Mass-Conserving Reaction-Diffusion Dynamics, Phys. Rev. X 10, 041036 (2020).

[26] M. E. Cates, D. Marenduzzo, I. Pagonabarraga, and J. Tailleur, Arrested phase separation in reproducing bacteria creates a generic route to pattern formation, Proc. Natl. Acad. Sci. U.S.A. 107, 11715 (2010).

[27] M.E. Cates and J. Tailleur, Motility-induced phase separation, Annu. Rev. Condens. Matter Phys. 6, 219 (2015).

[28] B. Liebchen and D. Levis, Collective Behavior of Chiral Active Matter: Pattern Formation and Enhanced Flocking, Phys. Rev. Lett. 119, 058002 (2017).

[29] H. Chaté, Dry aligning dilute active matter, Annu. Rev. Condens. Matter Phys. 11, 189 (2020).

[30] We discuss the dispersion relation of Eq. (1) linearized around a homogeneous steady state in Fig. S1 in Supplemental Material [32].

[31] Our findings immediately generalize to systems with density-independent cross-diffusion; see Supplemental Material Sec. 1.2 [32].

[32] See Supplemental Material at http://link.aps.org/ supplemental/10.1103/PhysRevLett.126.104101 for Movies $\mathrm{S} 1$ and $\mathrm{S} 2$ as well as technical background information, which includes Refs. [33-46].

[33] L. Onsager, Reciprocal relations in irreversible processes. I., Phys. Rev. 37, 405 (1931).

[34] P. C. Hohenberg and B.I. Halperin, Theory of dynamic critical phenomena, Rev. Mod. Phys. 49, 435 (1977).

[35] A. J. Bray, Theory of phase-ordering kinetics, Adv. Phys. 51, 481 (2002).

[36] N. D. Alikakos, G. Fusco, and G. Karali, Ostwald ripening in two dimensions - the rigorous derivation of the equations from the Mullins-Sekerka dynamics, J. Diff. Equations 205, 1 (2004).

[37] P. C. Bressloff, Two-dimensional droplet ripening in a concentration gradient, J. Phys. A 53, 365002 (2020).

[38] M. Tateno and S. Ishihara, Surface-tensiondriven coarsening in mass-conserved reaction-diffusion systems, arXiv:2010.03900.

[39] J. Rubinstein and P. Sternberg, Nonlocal reaction-diffusion equations and nucleation, IMA J. Appl. Math. 48, 249 (1992).

[40] I. S. Aranson, B. Meerson, P. V. Sasorov, and V. M. Vinokur, Phase Separation and Coarsening in Electrostatically Driven Granular Media, Phys. Rev. Lett. 88, 204301 (2002).

[41] L. Edelstein-Keshet, W. R. Holmes, M. Zajac, and M. Dutot, From simple to detailed models for cell polarization, Phil. Trans. R. Soc. B 368, 20130003 (2013).

[42] R. Diegmiller, H. Montanelli, C. B. Muratov, and S. Y. Shvartsman, Spherical caps in cell polarization, Biophys. J. 115, 26 (2018).

[43] A. S. Mikhailov, Foundations of Synergetics I: Distributed Active Systems (Springer, Berlin, 1990).
[44] D. Zwicker, R. Seyboldt, C. A. Weber, A. A. Hyman, and F. Jülicher, Growth and division of active droplets provides a model for protocells, Nat. Phys. 13, 408 (2017).

[45] Y. Shin and C. P. Brangwynne, Liquid phase condensation in cell physiology and disease, Science 357, eaaf4382 (2017).

[46] E. Gomes and J. Shorter, The molecular language of membraneless organelles, J. Biol. Chem. 294, 7115 (2019).

[47] Diffusive flux balance ensures that any stationary pattern can be dissected by inserting no-flux boundaries at its extrema.

[48] K. B. Glasner and T. P. Witelski, Coarsening dynamics of dewetting films, Phys. Rev. E 67, 016302 (2003).

[49] L. M. Pismen and Y. Pomeau, Mobility and interactions of weakly nonwetting droplets, Phys. Fluids 16, 2604 (2004).

[50] For mesa patterns in $1 \mathrm{D}, \partial_{M} \eta_{\text {stat }}$ must be calculated for the high- and low-density plateaus separately (see Supplemental Material Sec. 5 [32]).

[51] For a back of the envelope calculation, we use the density at the pattern inflection point $u_{0}$ and the interface width approximation $\ell_{\text {int }} \approx \pi /\left.q_{\max }\right|_{u_{0}, \eta_{\mathrm{stat}}}$ [25] and $\hat{u} \approx 2 u_{0}$ to estimate the peak mass $M=\int d x\left[\tilde{\rho}(x)-\rho_{-}\right] \approx \hat{u} \cdot \ell_{\text {int }} \approx$ $u_{0} \cdot 2 \pi /\left.q_{\max }\right|_{\eta_{\text {stat }}} \approx 2 \pi u_{0} \sqrt{D_{u} /\left.f_{u}\right|_{\eta_{\text {stat }}}}$. For the example $f_{\text {ex }}$, one finds $f_{u} \approx \eta_{\text {stat }}$ and $u_{0} \approx \eta_{\text {stat }}^{-1}$, and, thus, $M \sim \eta_{\text {stat }}^{-3 / 2}=$ $\eta_{\text {stat }}^{-1 / \alpha}$, for sufficiently large $M$ and $D_{v} \gg D_{u}$. Other reaction terms, e.g., with different nonlinearities in the recruitment term, yield other exponents $\alpha$.

[52] If $u, v$ describe (shifted) concentrations ( $u, v$ bounded from below) and $D_{u} / D_{v}$ is finite, mesas inevitably form at high densities [cf. Fig. 2(a)]. Arbitrarily large peaks form if no third FBS-NC intersection point exists, as, for example, in "model II" in Ref. [13]. The peak-to-mesa transition has previously been observed for unstable thin films subject to gravity [71].

[53] J. Langer, Theory of spinodal decomposition in alloys, Ann. Phys. (Leipzig) 65, 53 (1971).

[54] T. Kolokolnikov, T. Erneux, and J. Wei, Mesa-type patterns in the one-dimensional Brusselator and their stability, Physica (Amsterdam) 214D, 63 (2006).

[55] T. Kolokolnikov, M. Ward, and J. Wei, Selfreplication of mesa patterns in reaction-diffusion systems, Physica (Amsterdam) 236D, 104 (2007).

[56] In steady state, net degradation in high-density regions $\left(\rho>\rho_{\mathrm{HSS}}\right)$ and net production in low-density regions $\left(\rho<\rho_{\text {HSS }}\right)$ must balance.

[57] I. Prigogine and R. Lefever, Symmetry breaking instabilities in dissipative systems. II, J. Chem. Phys. 48, 1695 (1968).

[58] R. McKay and T. Kolokolnikov, Stability transitions and dynamics of mesa patterns near the shadow limit of reactiondiffusion systems in one space dimension, Discrete Contin. Dyn. Syst. B 17, 191 (2012).

[59] E. Tjhung, C. Nardini, and M. E. Cates, Cluster Phases and Bubbly Phase Separation in Active Fluids: Reversal of the Ostwald Process, Phys. Rev. X 8, 031080 (2018).

[60] V. K. Vanag and I. R. Epstein, Crossdiffusion and pattern formation in reaction-diffusion systems, Phys. Chem. Chem. Phys. 11, 897 (2009). 
[61] F. Rossi, V. K. Vanag, and I. R. Epstein, Pentanary crossdiffusion in water-in-oil microemulsions loaded with two components of the Belousov-Zhabotinsky reaction, Chemistry 17, 2138 (2011).

[62] A. Giri, S. Pramod Jain, and S. Kar, Alteration in cross diffusivities governs the nature and dynamics of spatiotemporal pattern formation, Chem. Phys. Chem. 21, 1608 (2020).

[63] G. Giunta, H. Seyed-Allaei, and U. Gerland, Cross-diffusion induced patterns for a single-step enzymatic reaction, Commun. Phys. 3, 167 (2020).

[64] Q.-X. Liu, A. Doelman, V. Rottschafer, M. de Jager, P. M. J. Herman, M. Rietkerk, and J. van de Koppel, Phase separation explains a new class of self-organized spatial patterns in ecological systems, Proc. Natl. Acad. Sci. U.S.A. 110, 11905 (2013).

[65] R. Wittkowski, A. Tiribocchi, J. Stenhammar, R. J. Allen, D. Marenduzzo, and M. E. Cates, Scalar $\Phi^{4}$ field theory for activeparticle phase separation, Nat. Commun. 5, 4351 (2014).
[66] G. Gonnella, D. Marenduzzo, A. Suma, and A. Tiribocchi, Motility-induced phase separation and coarsening in active matter, C. R. Phys. 16, 316 (2015).

[67] S. Sabrina, M. Spellings, S. C. Glotzer, and K. J. M. Bishop, Coarsening dynamics of binary liquids with active rotation, Soft Matter 11, 8409 (2015).

[68] G. Liu, A. Patch, F. Bahar, D. Yllanes, R. D. Welch, M. C. Marchetti, S. Thutupalli, and J. W. Shaevitz, Self-Driven Phase Transitions Drive Myxococcus xanthus Fruiting Body Formation, Phys. Rev. Lett. 122, 248102 (2019).

[69] A. I. Curatolo, N. Zhou, Y. Zhao, C. Liu, A. Daerr, J. Tailleur, and J. Huang, Engineering cooperative patterns in multi-species bacterial colonies, bioRxiv, https://doi.org/ 10.1101/798827 (2019).

[70] Y. I. Li and M. E. Cates, Non-equilibrium phase separation with reactions: A canonical model and its behaviour, J. Stat. Mech. (2020) 053206.

[71] M. B. Gratton and T. P. Witelski, Coarsening of unstable thin films subject to gravity, Phys. Rev. E 77, 016301 (2008). 